\title{
A Moment-Based Method of Optinalysis
}

\author{
Kabir Bindawa Abdullahi \\ Department of Biology, Faculty of Natural and Applied Sciences, \\ Umaru Musa Yar'adua University, Katsina-Nigeria \\ *Corresponding author's email: Kabir.abdullahi@umyu.edu.ng; kabirnamallam@gmail.com
}

\begin{abstract}
Graph symmetry detection, similarity, and identity measures have been extensively studied in graph automorphism and isomorphism problems. Nevertheless, graph isomorphism and automorphism detection remain an open (unsolved) problem for many decades. In this paper, a new optinalytic coefficient termed as an optical moment coefficient was introduced for optinalysis. Its characteristic efficiency was tested for bijective property, invariance, deterministic polynomiality and non-polynomiality. The test results show that the new optical moment coefficient is very efficient for symmetry detections, similarity and identity measures between two isometric isomorphs and automorphs; and deterministic on polynomial and nonpolynomial graph models.
\end{abstract}

Keywords: Automorphism; Identity; Isometry; Isomorphism; Similarity; Symmetry.

\section{Introduction}

The notion of isometry (as a congruence mapping) is a general phenomenon commonly accepted in Mathematics. It means a mapping that preserves distances. It is a bijective mapping, characterized as one-to-one mapping of a group onto itself or onto another in various transformational ways such as reflections, translation, or rotations (Miillman, R \& Parker, G, 1981).

Two graphs or sets are isomorphic if there is a bijection between their vertices or elements that preserves adjacency; such a bijection is called an isomorphism. In other terms, two graphs or sets $A$ and $B$ as isomorphic if they have the same structure, but their elements or vertices may be different. An isomorphism from a graph onto itself is called an automorphism, and the set of all automorphisms of a given graph $G$ denoted $\operatorname{Aut}(G)$, forms a group under composition (Hatori et al., 2006; Joyce, 2015).

The graph isomorphism problem is that of detecting the existence (or not) of an isomorphism between two input graphs. It is among a notorious problem in complexity theory, as no polynomial-time algorithm is known, and at the same time graph isomorphism is generally not believed to be NP-complete (Laszlo, 2016). Babai recently presented a quasipolynomial time algorithm for graph isomorphism (Oded et al., 1991). It is well-known that graph isomorphism is algorithmically and computationally polynomialtime equivalent (Ronald, C, and Derek, G 1977; Mathon 1979).

In applications, graph isomorphism has been applied to many structural entities from molecules to objects, and from music to physics, etc. For instance, several methods for detection of isomorphism problems in Kinematics such as Linkage Characteristic Polynomials Method, Hamming Number Technique, Degree Code Method, Link Adjacency Table Method, Distance Concept, Neural Network Approach, Fuzzy Logic Approach, Loop Based Detection Method, Genetic Algorithm Approach, Spanning Tree Method, Adjacency Matrix Method, Joint-Joint Matrix Method, etc have been proposed by many scholars from machine learning and algorithmic community (Arora \& Nigam, S, 2013; Shane et al., 2014). In addition to the polynomiality formalization, reliability of results, simplicity, applicability, detection of

Page 1 of 16 
inversions, etc are some of the definable attributes of efficient methods or algorithms in graph isomorphism detections (Arora \& Nigam, S, 2013; Shane et al., 2014). Until date, none of the existing methods is generally accepted to be efficient and a complete solution.

In this paper, an optical moment coefficient of optinalysis is introduced which looks at two finite graphs and sets as isometric isomorphs or automorphs as a mirror-like reflection of each other that expresses their degree of symmetry or identity and similarity. Optical moment coefficient of optinalysis is expressed in clearly defined terms and tested under some theorems and definitions of isometry, isomorphism, automorphism and optinalysis.

\section{Preliminary definitions and theorems}

Definition I. Injections, surjections, and bijections of functions between sets (Joyce, 2015).

These are words that describe certain functions $f: A \rightarrow B \rightarrow \mathrm{B}$ from one set to another.

An injection, also called a one-to-one function is a function that maps distinct elements to distinct elements, that is, if $x \neq y$, then $f(x) \neq f(y)$. Equivalently, if $f(x)=f(y)$ then, $x=y$.

$A$ surjection also called an onto function is one that includes all of $B$ in its image, that is, if $y \in B$, then there is an $x \in A$ such that $f(x)=y$.

$A$ bijection, also called a one-to-one correspondence, is a function that is simultaneously injective and bijective. Another way to describe a bijection $f: A \rightarrow B$ is to say that there is an inverse function $g$ : $B \rightarrow A$ so that the composition $g$ of $f: A \rightarrow A$ is the identity function on $A$ while $f$ o $g: B \rightarrow B$ is the identity function on $B$. The usual notation for the function inverse to $f$ is $f^{-1}$.

If $f$ and $g$ are inverse to each other, that is, if $g$ is the inverse of $f, g=f^{-1}$, then $f$ is the inverse of $g, f=g^{-1}$ Thus, $\left(f^{-1}\right)^{-1}=f$.

An important property of bijections is that you can convert equations involving $f$ to equations involving $f^{-1}$ :

$$
f(x)=y \text { if and only if } x=f^{-1}(y) .
$$

Definition II. Isometry (or congruence or congruent transformation) is a distance-preserving transformation between metric spaces, usually assumed to be bijective. Let $A$ and $B$ be metric space with metrics $d_{A}$ and $d_{B}$. A map $f: A \rightarrow B$ is called an isometry or distance preserving if for any $a, b \in A$ one has

$$
d_{B}\left(f(a), f(b)=d_{A}(a, b)\right.
$$

(Miillman, R \& Parker, G, 1981; Joyce, 2015).

Definition III. Isomorphism is a vertex bijection that preserves the adjacency or mathematical structures (e.g, vertices, edges, and non-edges) between two spaces sets and graphs that can be reversed by inverse mapping. Two mathematical structures $A$ and $B$ are isomorphic if they have the same structure, but their elements may be different (Hatori et al., 2006; Joyce, 2015).

$$
\begin{gathered}
f: A \rightarrow B \\
A \cong B
\end{gathered}
$$


Definition IV. Automorphism is an isomorphism from a mathematical object to itself. It is, in some sense; define the symmetry of the object, and a way of mapping the object to itself while preserving all of its mathematical structure (e.g vertices, edges, and non-edges) (Hatori et al., 2006; Joyce, 2015).

$$
\begin{gathered}
f: A \rightarrow \operatorname{Aut}\left(A^{\prime}\right) \\
A \cong A^{\prime}
\end{gathered}
$$

Definition $\boldsymbol{V}$. Scale can be defined as the system of marks at fixed intervals, which define the relationship between the units being used and their representation on the graph.

Definition VI. Optinalysis is a function that automorphically or isomorphically compares the symmetry, similarity, and identity between two spaces, graphs, and sets as a mirror-like (optic-like) reflection of each other about a symmetrical line or mid-point (Abdullahi, 2020).

Definition VII. Optinalysis is a function that comprised of an assigned optical (mirror) scale $(R)$ that bijectively re-maps $(\rightarrow)$ isometric and isomorphic or automorphic spaces, sets, and graphs under reflection about a symmetrical line or mid-point $(\delta)$. Figure 1 illustrates how two isometric isomorphs or automorphs are mapped and also re-mapped by an optical scale (Abdullahi, 2020).

Definition VIII. In comparative optinalysis, a reflection (pairing) is said to be head-to-head if the first labeled elements of two graphs, sets are the terminal points of the reflecting isometric isomorphs (Abdullahi, 2020).

$$
A=\left(A_{1}, A_{2}, A_{3}, \ldots \ldots, A_{n}\right) \underset{\mathrm{m}}{\delta} \quad B=\left(B_{n}, \ldots \ldots, B_{3}, B_{2}, B_{1}\right)
$$

Definition IX. In comparative optinalysis, a reflection or pairing is said to be tail-to-tail if the last labeled elements of graphs, sets are the terminal point of the reflecting isometric isomorphs (Abdullahi, 2020).

$$
A=\left(A_{n}, \ldots \ldots, A_{3}, A_{2}, A_{1}\right) \underset{\mathrm{m}}{\delta} \quad B=\left(B_{1}, B_{2}, B_{3}, \ldots \ldots, B_{n}\right)
$$

Theorem I. Isometric and isomorphic or automorphic spaces, sets and graphs are symmetrical or identical and similar to a certain magnitude by a coefficient (Abdullahi, 2020).

\section{Optinalytic Constructions and Definitions in Moment-Based Method of Optinalysis}

\subsection{Optinalytic constructions in moment-based method of optinalysis}

\subsubsection{Optinalytic constructions in general sense in the new optical scale}

Following this definition of optinalysis in box:

\section{Definition}

Optinalysis is a function that comprised of an assigned optical (mirror) scale $(R)$ that bijectively re-maps $(\rightarrow)$ isometric and isomorphic or automorphic spaces, sets, and graphs under reflection about a symmetrical line or mid-point $(\delta)$. Figure 1 illustrates how two isometric isomorphs or automorphs are mapped and also re-mapped by an optical scale (Abdullahi, 2020).

We can establish the optinalytic constructions as in Figure 1, which illustrates how two isometric isomorphs or automorphs are bijectively mapped and also bijectively re-mapped by the new optical scale. 


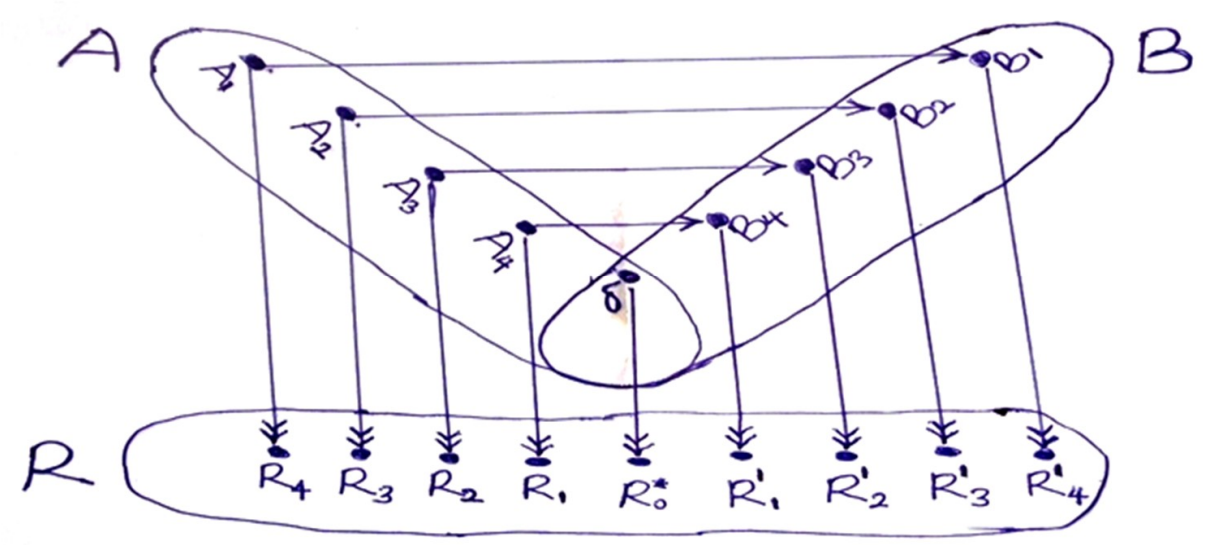

Figure I: Mapping between two isometric isomorphs or automorphs and re-mapping with the optical scale. $A$ is a domain; $B$ is a co-domain; $\delta$ is a mid-point or symmetrical line, and $R$ is an optical scale. The symbol $\rightarrow$ indicates a bijective mapping between the isometric isomorphs around a centre (midpoint) and $\rightarrow$ indicates a bijective re-mapping by the optical scale.

\subsubsection{The optinalytic construction in automorphic optinalysis}

It is expressed as:

$$
\begin{aligned}
& f: A \underset{m}{\stackrel{\delta}{\leftrightarrow}} A^{\prime} \rightarrow R
\end{aligned}
$$

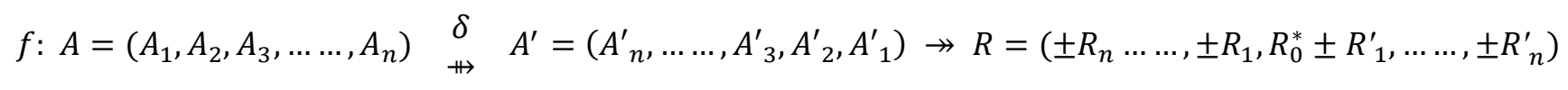

$$
\begin{aligned}
& f:\left[\begin{array}{ccc}
A=\left(A_{1}, A_{2}, A_{3}\right) & \delta & A^{\prime}=\left(A^{\prime}{ }_{3}, A^{\prime}{ }_{2}, A^{\prime}{ }_{1}\right) \\
\Downarrow & \stackrel{\#}{ } & \pm \\
R=\left( \pm R_{3}, \pm R_{2}, \pm R_{1}\right) & \pm R_{0}^{*} & \left( \pm R_{1}^{\prime}, \pm R^{\prime}{ }_{3}, \pm R_{3}^{\prime}\right)
\end{array}\right]
\end{aligned}
$$

Such that: $\left(A_{1}, A_{2}, A_{3}\right) \in A ;\left(A^{\prime}{ }_{1}, A^{\prime}{ }_{2}, A^{\prime}{ }_{3}\right) \in A^{\prime} ; \delta \in A \& A^{\prime} ; A, A^{\prime \prime} \& R \in \mathbb{R}, \mathbb{R}^{\mathrm{m}} ; R_{1} \neq 0$; and $A \& B$ are isometric automorphs of a named space, sets, and graphs.

\subsubsection{The optinalysis construction in isomorphic optinalysis}

It is expressed as:

$$
f: A \underset{\Perp}{\delta} \quad B \rightarrow R
$$

$$
\begin{aligned}
& f: A=\left(A_{1}, A_{2}, A_{3}, \ldots \ldots, A_{n}\right) \underset{\rightarrow}{\delta} \quad B=\left(B_{n}, \ldots \ldots, B_{3}, B_{2}, B_{1}\right) \rightarrow R=\left( \pm R_{n} \ldots \ldots, \pm R_{1}, R_{0}^{*} \pm R_{1}^{\prime}, \ldots \ldots, \pm R_{n}^{\prime}\right) \\
& f:\left[\begin{array}{ccc}
A=\left(A_{1}, A_{2}, A_{3}\right) & \delta & B=\left(B_{3}, B_{2}, B_{1}\right) \\
\Downarrow & \stackrel{\leftrightarrow}{ } & \downarrow \\
R=\left( \pm R_{3}, \pm R_{2}, \pm R_{1}\right) & \pm R_{0}^{*} & \left( \pm R_{1}^{\prime}, \pm R_{3}^{\prime}, \pm R_{3}^{\prime}\right)
\end{array}\right]
\end{aligned}
$$

Such that: $\left(A_{1}, A_{2}, A_{3}\right) \in A ;\left(B_{1}, B_{2}, B_{3}\right) \in B ; \delta \notin A \& B ; A, B \& R \in \mathbb{R}, \mathbb{R}^{\mathrm{m}} ; R_{1} \neq 0$; and $A \& B$ are isometric isomorphs of a named space, sets, and graphs. 


\subsection{Definitions in Moment-Based Method of Optinalysis}

Optinalysis is a function that bijectively maps optical nodes $(\mu)$ to their corresponding optical nodes $\left(\mu^{\prime}\right)$ about a symmetrical line or mid-point $(\delta)$. An optical node is defined by its moment. A moment, is the product of any member of a set and its assigned optical scale.

\section{Definition by Automorphic Optinalysis}

Suppose we have an optinalytic construction of isometric and automorphic spaces, sets and graphs with an assigned optical scale $(\mathrm{R}=3,2,1,0,1,2,3)$ as follows:

$$
f:\left[\begin{array}{ccc}
A=\left(A_{1}, A_{2}, A_{3}\right) & \delta & A^{\prime}=\left(A_{3}^{\prime}, A_{2}^{\prime}, A^{\prime}{ }_{1}\right) \\
\downarrow & \Downarrow & \downarrow \\
R=(3,2,1) & 0 & (1,2,3)
\end{array}\right]
$$

Such that $A$ and $A^{\prime}$ are isometric isomorphs on head-to-head reflection about a centre $(\delta)$.

$$
\begin{aligned}
& f: \mu \underset{\Pi}{\delta} \mu^{\prime} \\
& \mu=A \times R, \quad \& \quad \mu^{\prime}=A^{\prime} \times R^{\prime} \\
& f:(A \times R) \stackrel{\delta}{\infty} \quad\left(A^{\prime} \times R^{\prime}\right)
\end{aligned}
$$

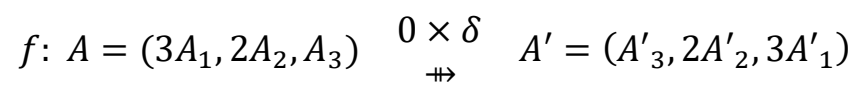

Definition by Isometric Optinalysis

Suppose we have an optinalytic construction of isometric and isomorphic spaces, sets and graphs with an assigned optical scale $(\mathrm{R}=3,2,1,0,1,2,3)$ as follows:

$$
f:\left[\begin{array}{ccc}
A=\left(A_{1}, A_{2}, A_{3}\right) & \delta & B=\left(B_{3}, B_{2}, B_{1}\right) \\
\Downarrow & \stackrel{\Downarrow}{\#} & \downarrow \\
R=(1,2,3) & 0 & (1,3,3)
\end{array}\right]
$$

Such that $A$ and $B$ are isometric isomorphs on head-to-head reflection about a centre $(\delta)$.

$$
\begin{aligned}
& f: \mu \underset{\mathbb{H}}{\delta} \mu^{\prime} \\
& \mu=A \times R \quad \& \quad \mu^{\prime}=B \times R^{\prime} \\
& f:(A \times R) \underset{\#}{\infty}\left(B \times R^{\prime}\right) \\
& f: A=\left(3 A_{1}, 2 A_{2}, A_{3}\right) \underset{\Pi}{0 \times \delta} \quad B=\left(B_{3}, 2 B_{2}, 3 B_{1}\right)
\end{aligned}
$$




\subsection{Optical moment coefficient of optinalysis}

An optical moment coefficient is expressed as the ratio of the sum of lowest optical moment to the sum of highest optical moment about a symmetrical line or midpoint. A valid result lies within a range $\geq$ $0 M_{c} \leq 1$.

Optical moment coefficient by shape optinalysis

Suppose we have an optinalytic construction of isometric and automorphic spaces, sets and graphs with an assigned optical scale $(\mathrm{R}=3,2,1,0,1,2,3)$ as follows:

$$
f:\left[\begin{array}{ccc}
A=\left(A_{1}, A_{2}, A_{3}\right) & \delta & A^{\prime}=\left(A^{\prime}, A^{\prime}{ }_{2}, A^{\prime}{ }_{1}\right) \\
\downarrow & \Downarrow & \Downarrow \\
R=(3,2,1) & 0 & (1,2,3)
\end{array}\right]
$$

Such that $A$ and $A^{\prime}$ are isometric automorphs on head-to-head reflection about a centre $(\delta)$.

Then, the Optical moment coefficient of symmetry or identity and similarity between the two isometric isomorphs is expressed as (Equations 1):

Let $\mu=\left(A_{n} \times R_{n}\right)$, or $\mu=\left(A_{n}^{\prime} \times R_{n}^{\prime}\right)$, be the moment about a centre.

$$
M_{c}=\frac{\sum_{1}^{n} \mu_{\text {smallest }}}{\sum_{1}^{n} \mu_{\text {largest }}}
$$

It can be expressed in percentage as:

$$
M_{c}=\frac{\sum_{1}^{n} \mu_{\text {smallest }}}{\sum_{1}^{n} \mu_{\text {largest }}} \times 100
$$

Such that each element is expressed as (Equations 1.1-1.6):

Suppose that $\mu=\sum_{1}^{n}\left(A_{n} \times R_{n}\right)<\mu=\sum_{1}^{n}\left(A_{n}^{\prime} \times R_{n}^{\prime}\right)$, then

$$
\begin{array}{r}
M_{c}=\frac{\sum_{1}^{n} \mu_{\text {smallest }}}{\sum_{1}^{n} \mu_{\text {largest }}}=\frac{A_{3}+2 A_{2}+3 A_{1}}{A^{\prime}{ }_{3}+2 A^{\prime}{ }_{2}+3 A^{\prime}}{ }_{1} \\
A_{1}=\frac{M_{c}\left(A^{\prime}{ }_{3}+2 A^{\prime}{ }_{2}+3 A^{\prime}{ }_{1}\right)-\left(2 A_{2}+A_{3}\right)}{3} \\
A_{2}=\frac{M_{c}\left(A^{\prime}{ }_{3}+2 A^{\prime}{ }_{2}+3 A^{\prime}{ }_{1}\right)-\left(3 A_{1}+A_{3}\right)}{2} \\
A_{3}=\frac{M_{c}\left(A^{\prime}{ }_{3}+2 A^{\prime}{ }_{2}+3 A^{\prime}{ }_{1}\right)-\left(3 A_{1}+2 A_{2}\right)}{1} \\
A^{\prime}{ }_{3}=\frac{M_{c}\left(2 A^{\prime}{ }_{2}+3 A^{\prime}{ }_{1}\right)-\left(3 A_{1}+2 A_{2}+A_{1}\right)}{M_{c}}
\end{array}
$$




$$
\begin{aligned}
& A^{\prime}{ }_{2}=\frac{M_{c}\left(A^{\prime}{ }_{3}+3 A^{\prime}{ }_{1}\right)-\left(3 A_{1}+2 A_{2}+A_{1}\right)}{2 M_{c}} \\
& A^{\prime}{ }_{1}=\frac{M_{c}\left(A^{\prime}{ }_{3}+2 A^{\prime}{ }_{2}\right)-\left(3 A_{1}+2 A_{2}+A_{1}\right)}{3 M_{c}}
\end{aligned}
$$

Optical moment coefficient by comparative optinalysis

Suppose we have an optinalytic construction of isometric and isomorphic spaces, sets and graphs with an assigned optical scale $(\mathrm{R}=3,2,1,0,1,2,3)$ as follows:

$$
f:\left[\begin{array}{ccc}
A=\left(A_{1}, A_{2}, A_{3}\right) & \delta & B=\left(B_{3}, B_{2}, B_{1}\right) \\
\Downarrow & \Downarrow & \downarrow \\
R=(3,2,1) & 0 & (1,2,3)
\end{array}\right]
$$

Such that $A$ and $B$ are isometric isomorphs on head-to-head reflection about a centre $(\delta)$.

Then, the Optical moment coefficient of symmetry or identity and similarity between the two isometric isomorphs is expressed as (Equations 2):

Let $\mu=\left(A_{n} \times R_{n}\right)$, or $\mu=\left(B_{n} \times R_{n}^{\prime}\right)$ be the moment about a centre.

$$
M_{c}=\frac{\sum_{1}^{n} \mu_{\text {smallest }}}{\sum_{1}^{n} \mu_{\text {largest }}}
$$

Such that each element is expressed as (Equations 2.1-2.6):

Suppose that $\mu=\sum_{1}^{n}\left(A_{n} \times R_{n}\right)<\mu=\sum_{1}^{n}\left(B_{n} \times R_{n}^{\prime}\right)$, then

$$
\begin{aligned}
& M_{c}=\frac{\sum_{1}^{n} \mu_{\text {smallest }}}{\sum_{1}^{n} \mu_{\text {largest }}}=\frac{A_{3}+2 A_{2}+3 A_{1}}{B_{3}+2 B_{2}+3 B_{1}} \\
A_{1}= & \frac{M_{c}\left(B_{3}+2 B_{2}+3 B_{1}\right)-\left(2 A_{2}+A_{3}\right)}{3} \\
A_{2}= & \frac{M_{c}\left(B_{3}+2 B_{2}+3 B_{1}\right)-\left(3 A_{1}+A_{3}\right)}{2} \\
A_{3}= & \frac{M_{c}\left(B_{3}+2 B_{2}+3 B_{1}\right)-\left(3 A_{1}+2 A_{2}\right)}{1} \\
B_{3}= & \frac{M_{c}\left(2 B_{2}+3 B_{1}\right)-\left(3 A_{1}+2 A_{2}+A_{1}\right)}{M_{c}} \\
B_{2}= & \frac{M_{c}\left(B_{3}+3 B_{1}\right)-\left(3 A_{1}+2 A_{2}+A_{1}\right)}{2 M_{c}} \\
B_{1}= & \frac{M_{c}\left(B_{3}+2 B_{2}\right)-\left(3 A_{1}+2 A_{2}+A_{1}\right)}{3 M_{c}}
\end{aligned}
$$




\subsection{Test for bijective mapping}

Optinalysis theorem: Pair of corresponding vertices under optinalysis are bijective (one-to-one and onto) to each other functionally (Abdullahi, 2020).

Recall the definition injective mapping (one-to-one), if $x=y$, then $f(x)=f(y)$, or equivalently if $x \neq y$, then $f(x) \neq f(y)$. Based on our problem under evaluation, and about equations 1.2 to 1.7 , and 2.1 to 2.7 of theorem 1 , we now have

Automorphically and isomorphically:

$$
\begin{aligned}
& \text { if } A_{1}=A^{\prime}{ }_{1} \text {, then } f\left(A_{1}\right)=f\left(A^{\prime}{ }_{1}\right) \text { or equivalently, if } A_{1} \neq A_{1}^{\prime} \text {, then } f\left(A_{1}\right) \neq f\left(B_{1}\right) \\
& \text { if } A_{2}=A^{\prime}{ }_{2} \text {, then } f\left(A_{2}\right)=f\left(A^{\prime}{ }_{2}\right) \text { or equivalently, if } A_{2} \neq A^{\prime}{ }_{2} \text {, then } f\left(A_{2}\right) \neq f\left(B_{2}\right) \\
& \text { if } A_{3}=A^{\prime}{ }_{3} \text {, then } f\left(A_{3}\right)=f\left(A^{\prime}{ }_{3}\right) \text { or equivalently, if } A_{3} \neq A^{\prime}{ }_{3} \text {, then } f\left(A_{3}\right) \neq f\left(B_{3}\right)
\end{aligned}
$$

Recall the definition surjective mapping (onto itself or other), if $y \in A$, then there is an $x \in A$ such that $f(x)=y$. Based on our problem under evaluation, and about equations 1.2 to 1.7 , and 2.1 to 2.7 of theorem 1, we now have:

Isomorphically:

if $A_{1} \in A$, then there is an $A^{\prime}{ }_{1} \in A$ such that $f\left(A_{1}\right)=A^{\prime}{ }_{1}$

if $A_{2} \in A$, then there is an $A^{\prime}{ }_{2} \in A$ such that $f\left(A_{2}\right)=A^{\prime}{ }_{2}$

if $A_{3} \in A$, then there is an $A^{\prime}{ }_{3} \in A$ such that $f\left(A_{3}\right)=A^{\prime}{ }_{3}$

Isomorphically and equivalently:

if $A_{1} \in A$, then there is an $B_{1} \in B$ such that $f\left(A_{1}\right)=B_{1}$

if $A_{2} \in A$, then there is an $B_{2} \in B$ such that $f\left(A_{2}\right)=B_{2}$

if $A_{3} \in A$, then there is an $B_{3} \in B$ such that $f\left(A_{3}\right)=B_{3}$

Therefore, optinalysis is based on the paradigm of the bijective mapping of defined finite sets of isometric isomorphs or automorphs.

\subsection{The test for optinalytic invariance (I)}

Optinalysis theorem: A perfect symmetry or identity and similarity state between isometric and isomorphic groups remains invariant (stable) with $M_{c}=1$ under other transformations such as pericentral rotation (alternate reflection), central rotation (inversion), product translation, additive translation, optical scaling (Abdullahi, 2020).

Suppose we have an optinalytic construction of isometric and isomorphic spaces, sets and graphs with an assigned optical scale $(\mathrm{R}=3,2,1,0,1,2,3)$ as follows:

$$
f:\left[\begin{array}{ccc}
A=\left(x^{2}, x^{4}, x^{3}\right) & \delta & B=\left(x^{3}, x^{4}, x^{2}\right) \\
\downarrow & \stackrel{\rightarrow}{ \pm} & \downarrow \\
R=(3,2,1) & 0 & (1,2,3)
\end{array}\right]
$$


Such that $A$ and $B$ are isometric isomorphs on head-to-head reflection about a centre $(\delta)$.

Then,

$$
\begin{gathered}
M_{c}=\frac{\sum_{1}^{n} \mu_{\text {smallest }}}{\sum_{1}^{n} \mu_{\text {largest }}} \\
M_{c}=\frac{3 x^{2}+2 x^{4}+1 x^{3}}{3 x^{2}+2 x^{4}+1 x^{3}}=1
\end{gathered}
$$

Therefore, $A$ and $B$ are perfectly similar and identical.

\subsubsection{The test for optinalytic invariance under additive translation}

Let the optinalytic construction of prove 3 be considered, and let ' $a$ ' be a translation factor. The optinalytic construction becomes:

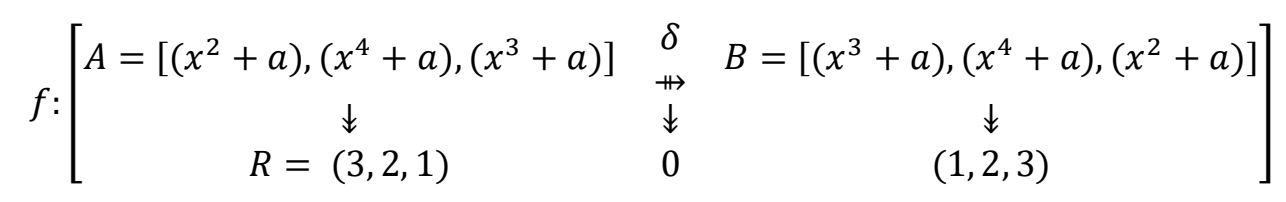

Such that $A$ and $B$ are isometric isomorphs on head-to-head reflection about a centre $(\delta)$.

Then,

$$
\begin{gathered}
M_{c}=\frac{\sum_{1}^{n} \mu_{\text {smallest }}}{\sum_{1}^{n} \mu_{\text {largest }}} \\
M_{c}=\frac{3\left(x^{2}+a\right)+2\left(x^{4}+a\right)+1\left(x^{3}+a\right)}{3\left(x^{2}+a\right)+2\left(x^{4}+a\right)+1\left(x^{3}+a\right)}=1
\end{gathered}
$$

Therefore, $A$ and $B$ are invariant under translation.

\subsubsection{The test for optinalytic invariance under product translation}

Let the optinalytic construction of prove 3 be considered, and let ' $a$ ' be a translation factor. The optinalytic construction becomes:

$$
f:\left[\begin{array}{ccc}
A=\left(a x^{2}, a x^{4}, a x^{3}\right) & \delta & B=\left(a x^{3}, a x^{4}, a x^{2}\right) \\
\downarrow & \stackrel{+}{\sharp} & \downarrow \\
R=(3,2,1) & 0 & (1,2,3)
\end{array}\right]
$$

Such that $A$ and $B$ are isometric isomorphs on head-to-head reflection about a centre $(\delta)$.

Then,

$$
\begin{gathered}
M_{c}=\frac{\sum_{1}^{n} \mu_{\text {smallest }}}{\sum_{1}^{n} \mu_{\text {largest }}} \\
M_{c}=\frac{3 a x^{2}+2 a x^{4}+1 a x^{3}}{3 a x^{2}+2 a x^{4}+1 a x^{3}}=1
\end{gathered}
$$

Page 9 of 16 
Therefore, $A$ and $B$ are invariant under translation.

\subsubsection{The test for optinalytic invariance under central rotation (Inversion)}

Let the optinalytic construction of prove 3 be considered, the centrally rotated structures becomes:

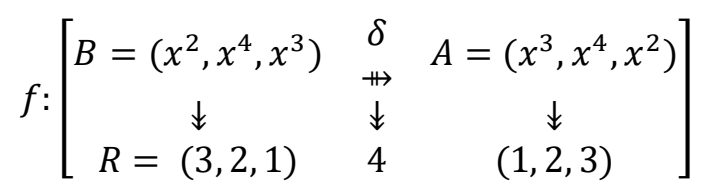

Such that $A$ and $B$ are isometric isomorphs on head-to-head reflection about a centre $(\delta)$.

Then,

$$
\begin{gathered}
M_{c}=\frac{\sum_{1}^{n} \mu_{\text {smallest }}}{\sum_{1}^{n} \mu_{\text {largest }}} \\
M_{c}=\frac{3 x^{2}+2 x^{4}+1 x^{3}}{3 x^{2}+2 x^{4}+1 x^{3}}=1
\end{gathered}
$$

Therefore, $A$ and $B$ are invariant under central rotation.

\subsubsection{The test for optinalytic invariance under pericentral rotation (Alternate reflection)}

Let the optinalytic construction of prove 3 be considered, the pericentrally rotated structures (inverses) of isometric and isomorphs becomes:

$$
f:\left[\begin{array}{ccc}
A=\left(x^{3}, x^{4}, x^{2}\right) & \delta & B=\left(x^{2}, x^{4}, x^{3}\right) \\
\Downarrow & \stackrel{+}{\downarrow} & \downarrow \\
R=(3,2,1) & 0 & (1,2,3)
\end{array}\right]
$$

Such that $A$ and $B$ are isometric isomorphs on tail-to-tail (its alternate) reflection about a centre $(\delta)$.

Then,

$$
\begin{gathered}
M_{c}=\frac{\sum_{1}^{n} \mu_{\text {smallest }}}{\sum_{1}^{n} \mu_{\text {largest }}} \\
M_{c}=\frac{3 x^{3}+2 x^{4}+1 x^{2}}{3 x^{3}+2 x^{4}+1 x^{2}}=1
\end{gathered}
$$

Therefore, $A$ and $B$ are invariant under pericentral rotation.

\subsubsection{The test for optinalytic invariance under optical scaling}

Let the optinalytic construction of prove 3 be considered, and let $R+a$ be the change in scaling patterns. The optinalytic construction becomes:

$$
f:\left[\begin{array}{ccc}
A=\left(x^{2}, x^{4}, x^{3}\right) & \delta & B=\left(x^{3}, x^{4}, x^{2}\right) \\
\downarrow & + & \ddagger \\
R=[(3+a),(2+a),(1+a)] & 0 & {[(1+a),(2+a),(3+a)]}
\end{array}\right]
$$


Such that $A$ and $B$ are isometric isomorphs on head-to-head reflection about a centre $(\delta)$.

Then,

$$
\begin{gathered}
M_{c}=\frac{\sum_{1}^{n} \mu_{\text {smallest }}}{\sum_{1}^{n} \mu_{\text {largest }}} \\
M_{c}=\frac{x^{2}(3+a)+x^{4}(2+a)+x^{3}(1+a)}{x^{2}(3+a)+x^{4}(2+a)+x^{3}(1+a)}=1
\end{gathered}
$$

Therefore, $A$ and $B$ are invariant under optical scaling.

\subsection{The test for optinalytic invariance under operations (II)}

Optinalysis theorem: Asymmetrical or dissimilar state between isometric and isomorphic or automorphic remains invariant (the same) under product translation, central rotation (inversion), and optical scaling (Abdullahi, 2020).

Suppose we have an optinalytic construction of isometric and isomorphic spaces, sets and graphs with an assigned optical scale $(\mathrm{R}=-3,-2,-1,0,-1,-2,-3)$ as follows:

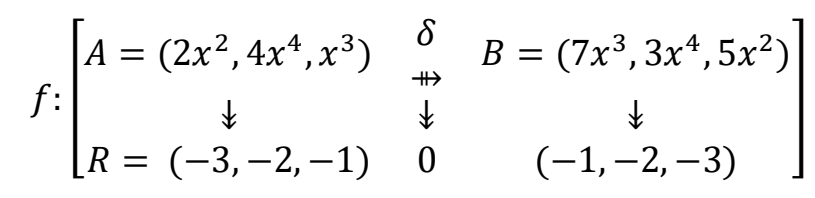

Such that $A$ and $B$ are isometric isomorphs on head-to-head reflection about a centre $(\delta)$.

Then,

$$
\begin{gathered}
M_{c}=\frac{\sum_{1}^{n} \mu_{\text {smallest }}}{\sum_{1}^{n} \mu_{\text {largest }}} \\
M_{c}=\frac{-6 x^{2}-8 x^{4}-x^{3}}{-7 x^{2}-6 x^{4}-15 x^{3}} \neq 1
\end{gathered}
$$

Therefore, $A$ and $B$ are dissimilar (asymmetrical).

\subsubsection{The test for optinalytic invariance under product translation}

Let the optinalytic construction of prove 4 be considered, and let ' $a$ ' be a translation factor. The optinalytic construction becomes:

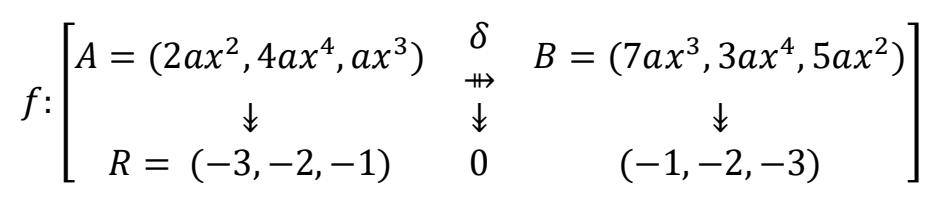

Such that $A$ and $B$ are isometric isomorphs on head-to-head reflection about a centre $(\delta)$.

Then, 


$$
\begin{gathered}
M_{c}=\frac{\sum_{1}^{n} \mu_{\text {smallest }}}{\sum_{1}^{n} \mu_{\text {largest }}} \\
M_{c}=\frac{-6 a x^{2}-8 a x^{4}-a x^{3}}{-7 a x^{2}-6 a x^{4}-15 a x^{3}} \neq 1
\end{gathered}
$$

Therefore, $A$ and $B$ are invariant under product translation.

\subsubsection{The test for optinalytic invariance under central rotation (Inversion)}

Let the optinalytic construction of prove 4 be considered, the centrally rotated structures of isometric isomorphs become:

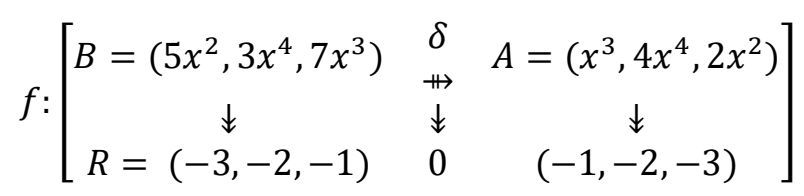

Such that $A$ and $B$ are isometric isomorphs on head-to-head reflection about a centre $(\delta)$.

Then,

$$
\begin{gathered}
M_{c}=\frac{\sum_{1}^{n} \mu_{\text {smallest }}}{\sum_{1}^{n} \mu_{\text {largest }}} \\
M_{c}=\frac{\left.-x^{3}-8 x^{4}-6 x^{2}\right)}{-15 x^{2}-6 x^{4}-7 x^{3}} \neq 1
\end{gathered}
$$

Therefore, $A$ and $B$ are invariant under product translation.

\subsubsection{The test for optinalytic invariance under optical scaling}

Let the optinalytic construction of prove 4 be considered, and let $(-R-a)$ be the change in scaling patterns. The optinalytic construction becomes:

$$
f:\left[\begin{array}{ccc}
A=\left(2 x^{2}, 4 x^{4}, x^{3}\right) & \delta & B=\left(7 x^{3}, 3 x^{4}, 5 x^{2}\right) \\
\downarrow & \stackrel{+}{*} & \downarrow \\
R=[(-3-a),(-2-a),(-1-a)] & 0 & {[(-1-a),(-3-a),(-3-a)]}
\end{array}\right]
$$

Such that $A$ and $B$ are isometric isomorphs on head-to-head reflection about a centre $(\delta)$.

Then,

$$
\begin{gathered}
M_{c}=\frac{\sum_{1}^{n} \mu_{\text {smallest }}}{\sum_{1}^{n} \mu_{\text {largest }}} \\
M_{c}=\frac{2 x^{2}(-3-a)+4 x^{4}(-2-a)+x^{3}(-1-a)}{7 x^{3}(-1-a)+3 x^{4}(-2-a)+5 x^{2}(-3-a)} \neq 1 \\
M_{c}=\frac{\left(-6 x^{2}-a\right)-\left(8 x^{4}-a\right)-\left(x^{3}-a\right)}{\left(7 x^{3}-a\right)-\left(6 x^{4}-a\right)-\left(15 x^{2}-a\right)} \neq 1
\end{gathered}
$$


Therefore, $A$ and $B$ are invariant under optical scaling.

\subsection{The test for optinalytic inverse equivalence}

Optinalysis theorem: The optical moment coefficients of optinalysis for inverse isometric isomorphs or automorphs remain the same as its former (Abdullahi, 2020).

$$
M_{c}:(A \underset{\leftrightarrow}{\delta} B \rightarrow R)=M_{C}:(B \underset{\leftrightarrow}{\delta} A \rightarrow R)
$$

Suppose we have an optinalytic construction as follows:

$$
f:\left[\begin{array}{ccc}
A=(2 x, 3 x, 6 x) & \delta=0 & B=(5 x, 3 x, x) \\
\downarrow & \stackrel{\Perp}{*} & \downarrow \\
R=(3,2,1) & 0 & (1,2,3)
\end{array}\right]
$$

Such that $A$ and $B$ are isometric isomorphs or automorphs on head-to-head reflection about a centre $(\delta)$.

Then,

$$
\begin{gathered}
M_{c}=\frac{\sum_{1}^{n} \mu_{\text {smallest }}}{\sum_{1}^{n} \mu_{\text {largest }}} \\
M_{c}=\frac{5 x+6 x+3 x}{6 x+6 x+6 x} \\
M_{c}=\frac{14 x}{18 x}=\frac{7 x}{9 x} \neq 1
\end{gathered}
$$

Inversely, the optinalytic construction becomes:

$$
f:\left[\begin{array}{ccc}
B=(x, 3 x, 5 x) & \delta=0 & A=(6 x, 3 x, 2 x) \\
\downarrow & \stackrel{m}{*} & \downarrow \\
R=(3,2,1) & 0 & (1,2,3)
\end{array}\right]
$$

Such that $A$ and $B$ are isometric isomorphs or automorphs on tail-to-tail reflection about a centre $(\delta)$.

Then,

$$
\begin{gathered}
M_{c}=\frac{\sum_{1}^{n} \mu_{\text {smallest }}}{\sum_{1}^{n} \mu_{\text {largest }}} \\
M_{c}=\frac{3 x+6 x+5 x}{6 x+6 x+6 x} \\
M_{c}=\frac{14 x}{18 x}=\frac{7 x}{9 x} \neq 1
\end{gathered}
$$

Now, the inverse equivalence by optical moment coefficient is 


$$
M_{c}:\left(A_{\rightarrow}^{\delta} B \rightarrow R\right)=M_{C}:(B \underset{m}{\delta} A \rightarrow R) \Rightarrow \frac{7 x}{9 x}=\frac{7 x}{9 x}
$$

Therefore, the inverse equivalences by percentage (probability) are equals to each other.

\subsection{The test for deterministic polynomiality and non-polynomiality}

Optinalytic theorem: Similarity, identity, and symmetry of isometric isomorphs and automorphs from named spaces, sets, and graphs are deterministic by optical moment coefficient on polynomial and nonpolynomial graph models, and also by the translated percentages or probabilities on polynomial and nonpolynomial graph models (Abdullahi, 2020).

Suppose we have a graph $A$ with a nodality of 20 vertices, and its single vertex iso-polymorphs $B$ were generated using an additive paranodic skewization approach, with a skewization value $t=10,25,50$, $10^{2}, 5 \times 10^{2}, 10^{3} \ldots \ldots 5 \times 10^{5}$ (Method adapted by (Abdullahi, 2019). The Optical moment coefficients and its valid translations were plotted graphically against its skewization value $t$ respectively.

$$
\begin{aligned}
& A_{(1-20)}=(25,28,15,5,55,10,33,12,70,90,45,64,23,87,67,34,97,45,34,78) . \\
& B_{1}=(\mathbf{2 5}+\mathbf{t}, 28,15,5,55,10,33,12,70,90,45,64,23,87,67,34,97,45,34,78) \\
& B_{2}=(25, \mathbf{2 8}+\mathbf{t}, 15,5,55,10,33,12,70,90,45,64,23,87,67,34,97,45,34,78) . \\
& B_{19}=(25,28,15,5,55,10,33,12,70,90,45,64,23,87,67,34,97,45,34+\mathbf{t}, 78) . \\
& B_{20}=(25,28,15,5,55,10,33,12,70,90,45,64,23,87,67,34,97,45,34,78+\mathbf{t}) .
\end{aligned}
$$

Let the optinalytic construction be defined as:

$$
f: A_{(1 \rightarrow 20)} \stackrel{\delta=0}{\rightarrow} \quad B_{(20 \rightarrow 1)} \rightarrow R=(20, \ldots 3,2,1,0,1,2,3, \ldots, 20)
$$

The optinalysis of these datasets was carried out using a customized Excel sheets presented in the supplementary material attached to this article.

Then, the optical moment coefficient between the graph $A_{1}$ and its iso-polymorphs $B_{(1 \rightarrow 20)}$ were obtained as $Q$.

Let $G$ be a set of positive integers that ranks the iso-polymorphic generations (from the first to the last) of iso-polymorphs established.

By plotting regression graphs of $G$ against $Q$, we observe a moving and changing regression patterns (from the best fits of linear/polynomial/exponential, continues as polynomial, shifts to logarithmic, and finally stagnates at power) as a skewization value $t$ approaches a certain maximum (See Fig. 2).

\section{Results}

The results present in Figures 2 reflect and justify the deterministic polynomiality and non-polynomiality of optinalytic theorem. 


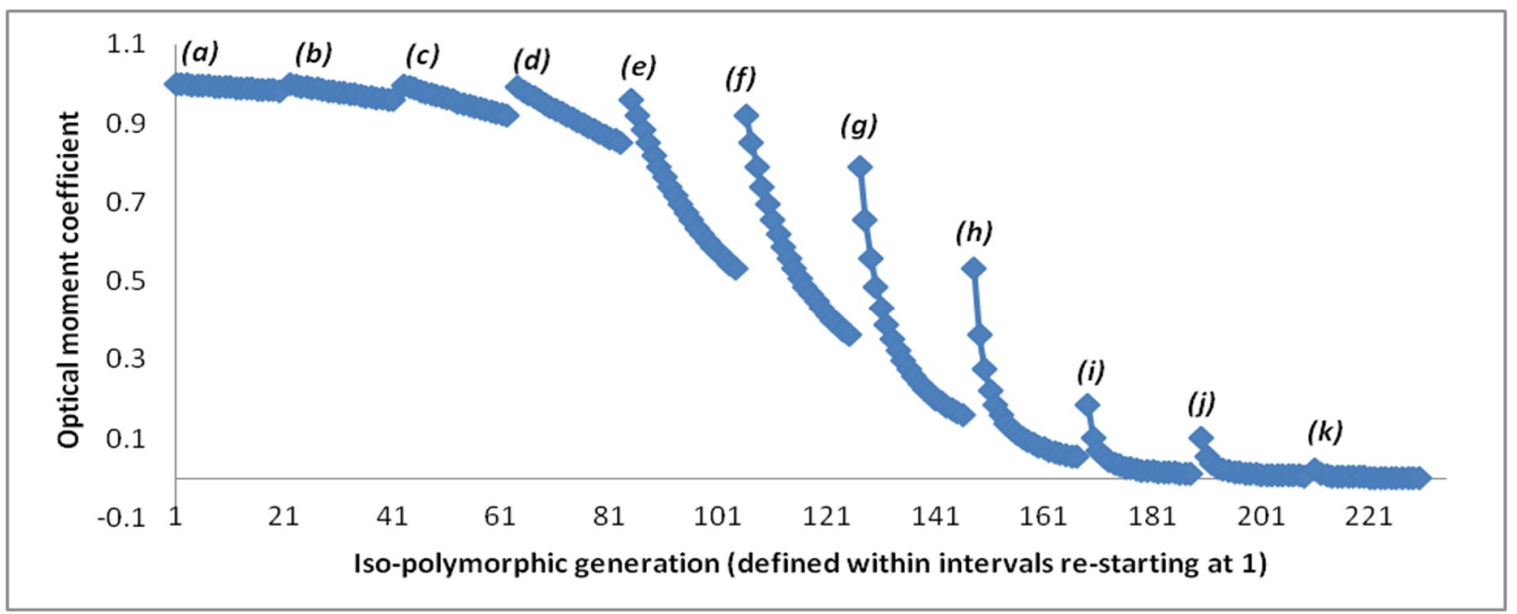

Figure 2: Graph models of the relationship between the generation number of iso-polymorphs and the resultant optical moment coefficient of optinalysis. (a)-(k) areee the graph models at skewization value $t=10,25,50$, $10^{2}, 5 \times 10^{2}, 10^{3}, 3 \times 10^{3}, 10^{4}, 5 \times 10^{4}, 10^{5}, 5 \times 10^{5}$ respectively. (a)-(d) are linear/polynomial/exponential, $(e-f)$ is polynomial, $(\boldsymbol{g})$ is logarithmic, and $(\boldsymbol{h})-(\boldsymbol{k})$ are power models.

Note: In this problem, the trending $M_{c}$ stagnates at a magnitude equal to zero.

\section{Discussion}

In this paper, a moment-based method of optinalysis for symmetry detections, similarity, and identity measures between two established isometric and isomorphic or automorphic graphs and sets is introduced. When we look at the optical scale, its uniform intervals preserve an equidistant relationship between the corresponding vertices or nodes of a named space, set, or graph. Furthermore, the optinalytic relationship between any pair of corresponding vertices is a clear bijection, which is also invertible, and these conform and translate the definitions and theorems of isometry, isomorphism, and automorphism.

When we look at the optical moment coefficient function, we tested that the results remain the same (invariant) under mathematical operations or transformations. This is a preservation of the mathematical structures of the isomorphs or automorphs. However, these properties of optical moment coefficient of optinalysis are sufficient evidences to prove its efficiency in graph symmetry detection, graphs similarity, and identity measures.

Concerning methodological efficiency, the polynomial and non-polynomial graph models generated by optical moment coefficient of optinalysis are very much the same as one of those generated by Kabirian coefficient of optinalysis (Abdullahi, 2020), and also very much analogous to the algorithmic formalization of polynomial-time of the effectiveness of computational methods of graph isomorphism problem. Therefore, optical moment coefficient of optinalysis is another complete solution to the graph isomorphism and automorphism problems in relation to symmetry detections, similarity and identity measures. This solution is analogous to Kabirian coefficient of optinalysis (Abdullahi, 2020), and unlike the recent quasipolynomial algorithm introduced by Babai (Laszlo, 2016).

\section{Conclusion}

Optical moment coefficient of optinalysis is an efficient moment-based method for symmetry detections, similarity, and identity measures between two established isometric and isomorphic or automorphic graphs and sets. The optical moment coefficient of optinalysis is based on isometry, isomorphism, and automorphism, expressed in clearly defined terms is tested to conform to the definitions and theorems of isometry, isomorphism, automorphism and optinalysis. Analogous to the polynomiality 
formalization of an efficient algorithm for graph isomorphism detections, optical moment coefficient of optinalysis is, however, deterministic on polynomial and non-polynomial graph models.

Supplementary material: A supplementary file S1 is an Excel customized sheets for a short-range test in optinalysis.

\section{Conflict of interest}

The author declares no conflict of interest.

Funding: This research did not receive any specific grant from funding agencies in the public, commercial, or not-for-profit sectors.

\section{References}

1. Abdullahi, K. B. (2019). Statistical Models in Comparative Optinalysis through Induced Systematic Skewization Mechanisms (Issue November). https://doi.org/10.20944/preprints201911.0141.v1

2. Abdullahi, K. B. (2020). Optinalysis : Isometric Isomorphism and Automorphism Through A Looking-Glass (Issue August). https://doi.org/10.20944/preprints202008.0072.v1

3. Arora, R., \& Nigam, S, P. (2013). A Comparative Study of Various Methods for Identification of Isomorphism in Kinematic Chains. Proceedings of the 1st International and 16th National Conference on Machines and Mechanisms (INaCoMM2013), 582-589.

4. Hatori, O., Miura, T., \& Takagi, H. (2006). Characterizations of isometric isomorphisms between uniform algebras via nonlinear range-preserving properties. 134(10), 2923-2930.

5. Joyce, D. (2015). Isomorphisms: Math 130 Linear Algebra. http://math.clarku.edu/ ma130/

6. Laszlo, B. (2016). Graph isomorphism in quasipolynomial time. In Proceedings of the 48th Annual ACM SIGACT Symposium on Theory of Computing, 684-697.

7. Mathon, R. (1979). A note on the graph isomorphism counting problem. Information Processing Letters, 8(3), 131-136.

8. Miillman, R, S., \& Parker, G, D. (1981). The Theory of Isometries. In: Geometry. Undergraduate Texts in Mathematics. Springer. https://doi.org/https://doi.org/10.1007/978-1-4684-0130-1_11

9. Oded, G., Silvio, M., \& Wigderson, A. (1991). Proofs that yield nothing but their validity or all languages in np have zero-knowledge proof systems. Journal of the ACM (JACM), 38(3), 690728.

10. Ronald, C, R., \& Derek, G, C. (1977). The graph isomorphism disease. Journal of Graph Theory, $1(4), 339-363$.

11. Shane, S., Rizvi, H., Hasan, A., \& Ph, D. (2014). A New Concept to Detect Isomorphism in Kinematic Chains using Fuzzy Similarity Index. 86(12), 30-33. 